\title{
Transarterial chemoembolization of hepatocellular carcinoma in a rat model: the effect of additional injection of survivin siRNA to the treatment protocol
}

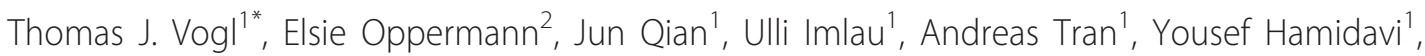
Huedayi Korkusuz', Wolf Otto Bechstein², Nour-Eldin Abdel-Rehim Nour-Eldin 1,3, Tatjana Gruber-Rouh', Renate Hammerstingl ${ }^{1}$ and Nagy Naguib Naeem Naguib ${ }^{1,4}$

\begin{abstract}
Background: Transarterial chemoembolization is one of the most widely accepted interventional treatment options for treatment of hepatocellular carcinoma. Still there is a lack of a standard protocol regarding the injected chemotherapeutics. Survivin is an inhibitor of Apoptosis protein that functions to inhibit apoptosis, promote proliferation, and enhance invasion. Survivin is selectively up-regulated in many human tumors. Small interfering RNA (siRNA) can trigger an RNA interference response in mammalian cells and induce strong inhibition of specific gene expression including Survivin. The aim of the study is to assess the effectiveness of the additional injection of Survivin siRNA to the routine protocol of Transarterial Chemoembolization (TACE) for the treatment of hepatocellular carcinoma in a rat model.

Methods: The study was performed on 20 male ACI rats. On day 0 a solid Morris Hepatoma 3924A was subcapsullary implanted in the liver. On day 12 MRI measurement of the initial tumor volume (V1) was performed. TACE was performed on day 13. The rats were divided into 2 groups; $\operatorname{Group}(A, n=10)$ in which $0.1 \mathrm{mg}$ mitomycin, $0.1 \mathrm{ml}$ lipiodol and $5.0 \mathrm{mg}$ degradable starch microspheres were injected in addition $2.5 \mathrm{nmol}$ survivin siRNA were injected. The same agents were injected in Group $\left(B_{1}=10\right)$ without Survivin siRNA. MRI was repeated on day 25 to assess the tumor volume (V2). The tumor growth ratio (V2N1) was calculated. Western blot and immunohistochemical analysis were performed.

Results: For group A the mean tumor growth ratio (V2N1) was $1.1313+/-0.1381$, and was $3.1911+/-0.1393$ in group B. A statistically significant difference between both groups was observed regarding the inhibition of tumor growth $(P<0$. 0001) where Group A showed more inhibition compared to Group B. Similarly immunohistochemical analysis showed significantly lower $(p<0.002)$ VEGF staining in group A compared to group B. Western Blot analysis showed a similar difference in VEGF expression $(P<0.0001)$.

Conclusion: The additional injection of Survivin siRNA to the routine TACE protocol increased the inhibition of the hepatocellular carcinoma growth in a rat animal model compared to regular TACE protocol.
\end{abstract}

Keywords: Hepatocellular carcinoma, Survivin siRNA, Chemoembolization

\footnotetext{
*Correspondence: T.Vog|@em.uni-frankfurt.de

'Institute for Diagnostic and Interventional Radiology, Johann Wolfgang

Goethe-University, Theodor-Stern-Kai 7, Frankfurt 60590, Germany

Full list of author information is available at the end of the article
} 


\section{Background}

Being one of the most common malignancies in the world Hepatocellular carcinoma (HCC) is estimated to be responsible for about one million deaths per year. Owing to its rapid infiltrating growth and complicating liver cirrhosis HCC usually have a poor prognosis [1]. Transarterial chemoembolization (TACE) is a widely accepted therapeutic option for HCC [2]. Survivin is an inhibitor of Apoptosis protein that functions to inhibit apoptosis, promote proliferation, and enhance invasion [3]. Under normal conditions Survivin is expressed in embryonic and fetal tissues and is barely detectable in most differentiated normal adult tissues [4]. In addition, it has been shown that Survivin expression is present in some normal adult tissue cells like T-Lymphocytes, polymorphonuclear-neutrophils, primitive hematopoietic cells and vascular endothelial cells $[5,6]$.

Survivin is selectively up-regulated in many human tumors, an overexpression of Survivin correlates with poor outcome, treatment resistance [3] reduced disease free survival and overall survival of cancer patients [7]. It has been shown to increase resistance of tumor tissue to apoptotic stimuli mainly through a Caspase-dependent mechanism [5]. Small interfering RNA (siRNA) can trigger an RNA interference response in mammalian cells and induce strong inhibition of specific gene expression hence they can be used to inhibit cancer-related genes including Survivin [8].

In-spite of the wide acceptance of TACE a meta-analysis [9] has demonstrated that 4 of the 6 randomized controlled studies included in the analysis comparing TACE with untreated controls failed to show any impact of TACE on patient survival. Keeping into consideration that there is a great controversy regarding the best chemotherapeutic agents for TACE [10] the need for introducing new chemotherapeutic agents targeting $\mathrm{HCC}$ is critical.

Thus, the aim of the current study was to assess the therapeutic efficacy of the additional transarterial injection of Survivin siRNA to the regular TACE protocol compared to the regular TACE protocol in an animal model of hepatocellular carcinoma, and its effect on tumor growth, angiogenesis and vascular endothelial growth factor expression (VEGF) levels.

\section{Methods}

\section{Animals and tumor cells}

All of the experiments on animals were approved by the Regional Administrative Authority in Darmstadt, Germany.

Twenty inbred male ACI-rats (Harlan Winkelmann; Borchen, Germany) weighing 220-240 g were used. The animals were kept under conventional conditions with a temperature of $22 \pm 2{ }^{\circ} \mathrm{C}$, a relative humidity of $55 \pm$ $10 \%$, a dark-light rhythm of $12 \mathrm{~h}$, and they were fed with standard laboratory chow and tap water ad libitum. The hepatoma cell line used in the current study was obtained from the German Cancer Research Center (DKFZ; Heidelberg, Germany). The injected cell line (Morris hepatoma 3924A) represents a rapidly growing, poorly differentiated hepatocellular carcinoma.

\section{Chemotherapeutics and agents}

For TACE a dose of $0.1 \mathrm{mg}$ Mitomycin (Roche, Grenzach-Wyhlen, Germany) was dissolved in $0.1 \mathrm{ml}$ $0.9 \% \mathrm{NaCl}$ solution $10 \mathrm{~min}$ before application. The embolization was performed using a dose of $0.1 \mathrm{ml}$ lipiodol (Guerbet $\mathrm{GmbH}$, Sulzbach, Germany) and $5.0 \mathrm{mg}$ degradable starch Microspheres (Spherex®), Pharmacia, Erlangen, Germany).

Survivin siRNA was provided from Ruibo Gentech Co (Wuhan, PR. China). The target sequence of Survivin siRNA is as follows: CCGAGAATGAGCCTGATTT. A dose of $2.5 \mathrm{nmol}$ Survivin siRNA was stable at $2-8{ }^{\circ} \mathrm{C}$ for $10 \mathrm{~min}$ before administration.

\section{Anesthesia}

A combination of intra-peritoneal injection of Ketamine Hydrochloride (Ketanest Parke-Davis, Germany; $100 \mathrm{mg} / \mathrm{kg}$ ), Xylazine Hydrochloride (Rompun, Bayer Germany; $15 \mathrm{mg} / \mathrm{kg}$ ) and Atropine Sulfate (Atropin Sulfat Braun, Braun, Germany; $0.1 \mathrm{mg} / \mathrm{kg}$ ) was used for anesthesia in all Orthotopic, interventional and imaging procedures.

\section{Tumor implantation (day 0)}

The technique for tumor implantation was basically similar to that described by Yang et al. [11] with minor modifications [12]. The Morris Hepatoma 3924A tumor tissue, recovered from the passaged animals 12 days after subcutaneous implantation (corresponding to $5 \times$ $10^{6}$ tumor cells), was cut into small cubes about $2 \mathrm{~mm}^{3}$. A small sub-capsular incision on the left lateral lobe of the liver was made in the recipient ACI-rats under anesthesia. The tumor fragment was gently placed into created pocket with a small cotton swab on the liver surface and the abdominal wall was then closed.

\section{Interventional therapy (day 13)}

For interventional studies a second laparotomy was performed. By using a binocular operative microscope (M651, Leica; Wetzler, Germany), a PE-10 polyethylene Micro-catheter (inner diameter $0.28 \mathrm{~mm}$, outer diameter $0.61 \mathrm{~mm}$; Wenzel; Heidelberg, Germany) was retrogradely inserted into the Gastro-duodenal artery and pushed forward to the hepatic artery. Different agents were then injected into the hepatic artery using the sandwich technique (subsequent injection of Mitomycin 
+/- Survivin siRNA + Lipiodol + degradable starch Microspheres) within $20 \mathrm{~min}$. Each group of animals received treatment according to the following protocols:

- Group A (TACE + Survivin siRNA, test group, $n=10$ ): Mitomycin (0.1 mg) + Lipiodol (0.1 ml) + degradable starch Microspheres $(5.0 \mathrm{mg})+$ Survivin siRNA $(2.5 \mathrm{nmol})$

- Group B (TACE alone, control group, $n=10$ ): Mitomycin $(0.1 \mathrm{mg})+$ Lipiodol $(0.1 \mathrm{ml})+$ degradable starch Microspheres $(5.0 \mathrm{mg})$

\section{MR imaging and analysis (day 12 and 25)}

MRI imaging before (on day 12) and after (on day 25) treatment was performed using a 3.0 Tesla MRI unit (Magnetom, Siemens; Erlangen, Germany) by using a wrist coil (Small field of view). T1-weighted (SE: TR/TE, 500/12 ms) and T2-weighted (TSE: TR/TE, 3870/80 ms) transverse images with a section thickness of $2 \mathrm{~mm}$ and $184 \times 256$ matrix were acquired. There was no gap between sections and no contrast medium was administered. The tumor volume was determined and evaluated in the T2-weighted image according to the formula [13]: $\mathrm{V}=0.5 \times \mathrm{d} 1 \times \mathrm{d}^{2}$, where $\mathrm{d} 1$ is the maximum diameter of the tumor and $\mathrm{d} 2$ is the minimum diameter perpendicular to d1. Image evaluations and size assessments were performed by a single radiologist with more than 15 years' experience in abdominal MRI imaging and who was blinded to the group assignment of the animal in the study.

\section{Western blot (day 25)}

Western blot analysis was carried out to determine the expression level of the VEGF in the two groups. After the MRI examination, all the rats were sacrificed using an over-dose of intravenous Sodium Pentobarbital. To homogenize the tumors, Precellys Homogenizer IV (Peqlab Biotechnologie GmbH; Erlangen, Germany) at $4{ }^{\circ} \mathrm{C}$ was used in a lysis buffer which is composed of $50 \mathrm{mM}$ HEPES, $200 \mathrm{mM} \mathrm{NaCl}, 0.2 \mathrm{mM}$ MgSO4, $0.4 \mathrm{mM}$ Phenylmethylsulfonyl fluoride, $2 \%$ Triton- X100, $10 \mu \mathrm{g} / \mathrm{mL}$ Leupeptine, $10 \mu \mathrm{g} / \mathrm{mL}$ Aprotinine, $0.02 \%$ soybean trypsin inhibitor and $0.2 \mathrm{mM}$ Orthovanadate (Sigma-Aldrich; Taufkirchen, Munich, Germany). The resulting Cell lysates were centrifuged for $10 \mathrm{~min}$ at $12,000 \times \mathrm{g}$ at $4{ }^{\circ} \mathrm{C}$. Coomassie Plus protein assay kit (Pierce; Rockford, IL, USA) was used to measure the protein concentration in the supernatants. The protein concentration results were obtained Spectrophotometrically by Tecan Infinite ${ }^{\circ}$ M 200 microplate reader (Tecan-Deutschland; Crailsheim, Germany) at $595 \mathrm{~nm}$. Protein was then denatured in Laemmli sample buffer (Bio-Rad Laboratories; Munich, Germany) with $\beta$ mercaptoethanol (Sigma; Taufkirchen, Germany), boiled for
$5 \mathrm{~min}$, and transferred on ice. Sodium Dodecyl Sulfate Polyacrylamide gel electrophoresis (SDS-PAGE) $(50 \mu \mathrm{g}$ per lane) was then conducted. The molecular weight standards used were PeqGold prestained protein markers IV (Peqlab Biotechnologie GmbH; Erlangen, Germany). After separation using gel electrophoresis, protein was blotted onto a Polyvinylidene Difluoride membrane (Hybond P; GE Healthcare; Munich, Germany). Blots were then blocked with $10 \%$ low-fat milk for $1 \mathrm{~h}$ at room temperature followed by overnight incubation at $4{ }^{\circ} \mathrm{C}$ with primary antibody from Santa Cruz Biotechnology (Rabbit polyclonal VEGF 1:200; Rabbit polyclonal MMP-9 and mouse monoclonal $\beta$ Actin, (clone AC-15, 1:1000; Sigma). Blots were then washed 3 times with Towbin buffer with $0.5 \%$ Tween 20 followed by incubation for $30 \mathrm{~min}$ at room temperature with secondary antibody from Millipore $\mathrm{GmbH}$; Schwalbach/Ts, Germany (Polyclonal goat Anti-rabbit IgG, 1:5000; goat Anti-mouse IgG, 1:5000, both HRP conjugated). All antibodies were diluted in Towbin Buffer with $0.5 \%$ Tween 20 and $0.5 \%$ bovine serum albumin. Blots were then washed and incubated withEnhanced Chemiluminescence detection kit (GE Healthcare; Munich, Germany). Signal intensity was finally detected and captured by Fusion FX-7 (Vilber Lourmat, Marnee la Vallee, France), documented and analyzed by Bio1D software (Vilber Lourmat). $\beta$ Actin was used as the loading control.

\section{Immunohistochemical examination (day 26)}

The Liver samples were embedded and frozen in a TissueTek (Sakura, Zoeterwoude, Netherlands) and $5 \mu \mathrm{M}$ cryosections were prepared. These Sections were fixed in $100 \%$ acetone and equilibrated in PBS followed by overnight incubation at $4{ }^{\circ} \mathrm{C}$ with anti-VEGF rabbit polyclonal antibody (Santa Cruz Biotechnology Inc., USA) which was diluted with Dako antibody diluents (DAKO, Hamburg, Germany). The cryosections were then incubated with Anti-rabbit Alkaline Phosphatase supervision polymer system (DCS Innovative Diagnostik-Systeme, Hamburg, Germany). Staining was visualized using the Neu Fuchsin substrate Chromogen (DCS Innovative DiagnostikSysteme, Hamburg, Germany) and were counterstained with Hematoxylin and mounted in Kaisers Glycerol Gelatin (Merck, Darmstadt, Germany). To evaluate the expression of VEGF, all slides were examined and scored by two independent pathologists who were blinded to the animal data. The percentage staining was scored as follows: 0 (No staining, 1 (0-5 \%), 2 (6-25 \%), 3 (26$50 \%), 4$ (51-75 \%), 5 (76-100 \%).

\section{Statistical analysis}

The mean tumor growth ratio (V2/V1, V2 tumor volume after treatment and V1 tumor volume before 
treatment) by MRI and the mean expression ratio (VEGF/ $\beta$-actin) level of VEGF by Western blot from each group were measured and the significance of differences between the two groups were analyzed using the paired-t-test, the statistical software used was GraphPad Prism (version 3.02, La Jolla, CA, USA). Immunohistochemical staining of VEGF was evaluated using descriptive and semi-quantitative methods. The differences between both groups in the Western-Blot analysis and Immunohistochemical analysis were tested for statistical significance using the unpaired-ttest and the Wilcoxon signed rank test respectively. Differences with a $\mathrm{p}$ value less than 0.05 were considered statistically significant.

\section{Results}

\section{MRI examination}

Tumor implantation was successful in $100 \%$ of the rats. Most tumors appeared homogeneous and were hypointense on T1-weighted images and hyperintense on T2weighted images prior to treatment, after treatment the tumors appeared inhomogenous. After different interventional treatments, intrahepatic metastases developed in two of the 10 rats in group B. The means of the volume ratios (V2/V1) were $1.1313 \pm 0.1381$ in group $A$, and $3.1911 \pm 0.1393$ in group B. Compared to group B, group A showed a statistically significant reduction of the tumor growth within the period of observation $(p<0.0001)$ (Fig. 1).

\section{Western blot analysis}

VEGF expression level was lower in group A (TACE + Survivin siRNA) than in group B (TACE alone). There was a statistically significant difference between group $A$ and group $\mathrm{B}$ regarding the VEGF expression by Western blot analysis $(p<0.0001)$ (Fig. 2).

\section{Immunohistochemical assay}

The angiogenesis of the tumor was evaluated using the Anti-VEGF antibodies. VEGF were expressed in all specimens. The Immuno-expression of the protein was confirmed by the presence of brown stained cytoplasm in tumor cells. Higher expression of VEGF in hepatocellular carcinoma was observed in group B (TACE alone) with a median histological score of 4.250 compared to group A (TACE + Survivin siRNA) with a median histological score of 2.450. The difference between both groups was statistically significant $(p=0.0020)$ (Table 1$)$ (Fig. 3).

\section{Discussion}

Since the introduction of TACE as a palliative treatment in patients with unresectable HCC, it has become one of the most common forms of interventional therapy $[2,10]$.

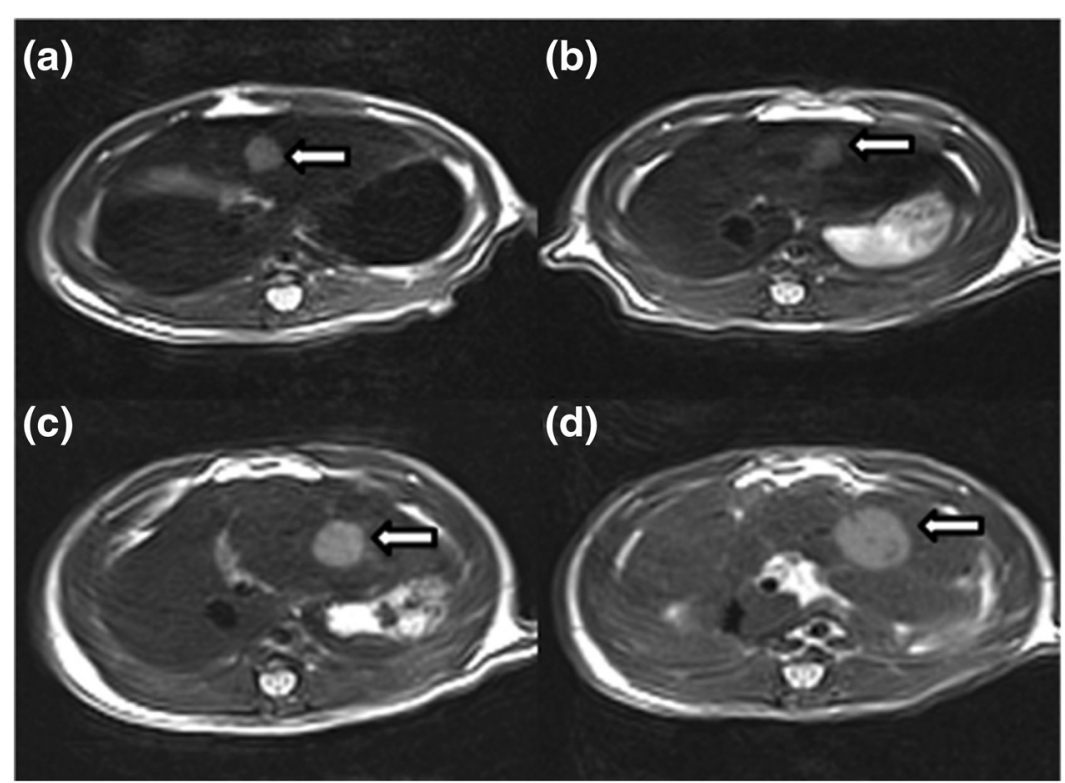

(e)

Mean tumor growth ratio

Fig. 1 Transverse unenhanced T2-weighted TSE MR images of solid liver tumor in a group A (TACE+ survivin siRNA) (images a and b) and group B (control group, TACE alone) (images C and d) in ACl rat. 3870/80 matrix was acquired. a Pretreatment shows a small hyperintense tumor (arrow) in the left lateral liver lobe $(0.55 \times 0.54 \mathrm{~cm})$. b Posttreatment demonstrates the same hyperintense tumor lesion (arrow) $(0.55 \times 0.53 \mathrm{~cm})$ and has inhomogeneous hypointense area corresponding to intratumoral necrosis. The growth of hepatic tumor is noticeably inhibited after therapy. $\mathbf{c}$ Pretreatment shows a small hyperintense tumor (arrow) in the left lateral liver lobe $(0.69 \times 0.68 \mathrm{~cm})$. $\mathbf{d}$ Posttreatment shows the same $(1.14 \times 0.98 \mathrm{~cm})$ tumor (arrow) exhibiting rapid growth compared with its size before therapy. e Mean tumor growth ratio of post-treated (V2) and pre-treated tumor (V1) by MRI showed significant difference between group A (TACE + survivin siRNA) vs group B (control group, TACE alone) 


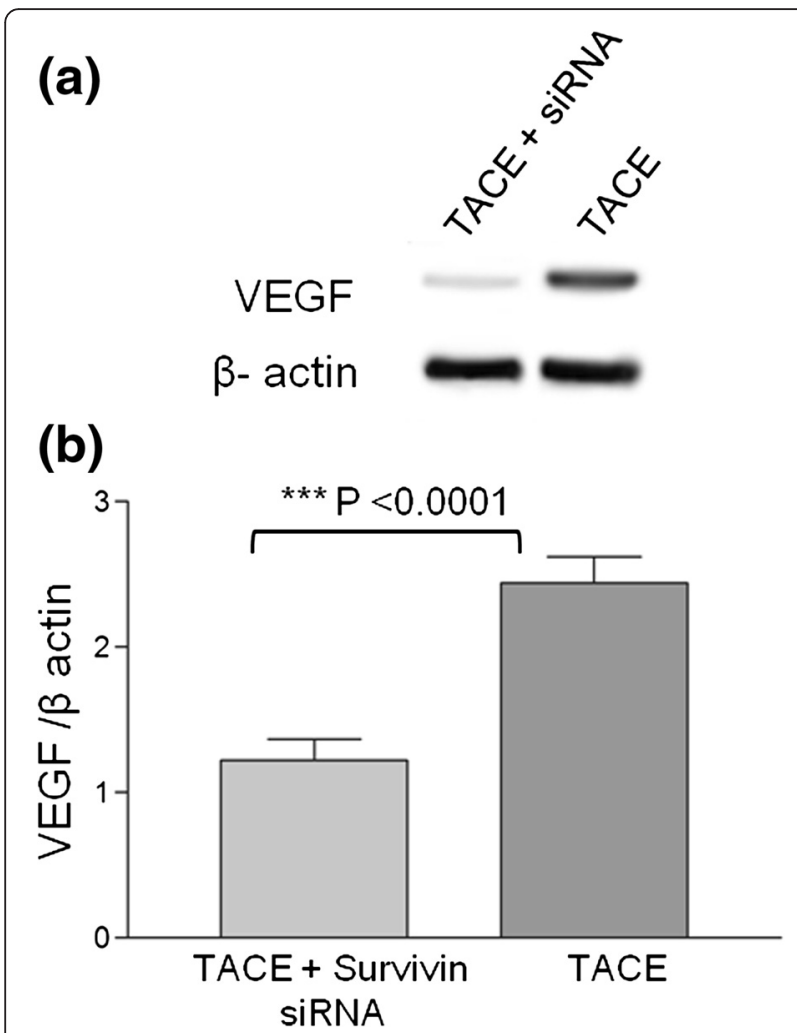

Fig. 2 Inhibitory effects of survivin siRNA on the expression of VEGF by Western blot analysis. a VEGF bands in group A (TACE + survivin siRNA) was suppressed compared to TACE alone (control). Depicted are representative bands from 10 independent experiments $(N=10)$ (b) Shows the semi quantification of proteins bands, expressed as ratio of VEGF / $\beta$-actin. Group A (TACE + survivin siRNA) was down regulated compared to Group B (TACE alone) $(N=10)$

TACE reduces the maximum plasma concentration, lengthens the half-life, and increases the average concentration of chemotherapeutic agents in the tumor [14]. However, post-interventional metastasis and recurrence of tumors have hindered the curative effect of interventional therapeutic procedures and the long-term survival rates [2]. Moreover, a study by Kobayashi et al. [15] showed that the serum concentration of VEGF was markedly increased in patients following embolization.

Biologically, Survivin has been shown to inhibit apoptosis, enhance proliferation and promote angiogenesis. High expression levels of Survivin correlate with an

Table 1 Immunuhistochemical expression of VEGF in hepatocellular carcinoma

\begin{tabular}{llll}
\hline Tumor & $\begin{array}{l}\text { Tace }+ \text { Survivin } \\
\text { siRNA }(N=10) \\
\text { Median Score }\end{array}$ & $\begin{array}{l}\text { TACE }(N=10) \\
\text { Median Score }\end{array}$ & $\begin{array}{l}P \text { value (Wilcoxon } \\
\text { signed rank test) }\end{array}$ \\
\hline VEGF & 2.450 & 4.250 & 0.0020 \\
\hline
\end{tabular}

Scoring: 0 (No staining), 1 (0-5\%), 2 (6-25\%), 3 (26-50\%), 4 (51-75\%), 5 (76-100\%). increased rate of tumor recurrence and resistance to chemotherapy [16]. Several in vitro and in vivo studies have indicated that Survivin down-regulation is able to sensitize human tumor cells of different histologic origins to conventional chemotherapeutic drugs [17]. Another important point to notice is that Survivin plays an important role in response to Radiotherapy too; a high level of Survivin has been shown to increase both the resistance to Radiotherapy and the incidence of local recurrence in rectal cancer patients [18] and is associated with worsened survival in patients treated with definitive Radiotherapy for cervical cancer [7, 19]. Similarly, it might be suggested that Survivin expression might have a similar effect on patient response to Radioembolization of hepatic malignancy and that inhibiting Survivin expression using Survivin siRNA might have a favorable effect on patient response to Radioembolization; similar to what was previously reported by Yang et al. [20] where the authors reported an enhanced radiosensitivity in human hepatoma cells both in vitro and in vivo in response to Survivin downregulation by Survivin siRNA. Hence combining Survivin siRNA and Radioembolization might be associated with an improved response to Radioembolization, still the medical literature is lacking such a study.

As Survivin is not a cell surface protein and does not have an intrinsic enzymatic activity, targeting of Survivin for therapeutic purposes might be expected to be difficult. In addition, crystallographic data has revealed few potential drug able sites on Survivin protein [21]. Because of the up-regulation of Survivin in malignancy and its key role in apoptosis, proliferation and angiogenesis, Survivin is currently attracting considerable attention as a new target for anti-cancer therapies. Strategies under investigation to target Survivin include antisense oligonucleotides, small interfering RNA (siRNA), ribozymes, immunotherapy and small molecular weight molecules [22].

Recently, siRNA technology holds a great promise as a therapeutic intervention for targeted gene silencing in cancer. RNA interference (RNAi) is a biological mechanism whereby the presence of double-stranded RNA (dsRNA) interferes with the expression of a particular gene that shares a homologous sequence with the dsRNA. Recent studies have provided insights into the molecular mechanisms of RNAi, in which dsRNA induces the silencing of homologous mRNA. In the cytoplasm of mammalian cells, an enzyme known as Dicer initiates RNA silencing by the breakdown of long dsRNA to generate siRNA of about 21-23 nucleotides in length. The resulting siRNAs are incorporated into an RNAinduced silencing complex (RISC) and unwound into a single-stranded RNA (ssRNA), which is followed by the degradation of ssRNA [23]. siRNA is 10-100-fold more 


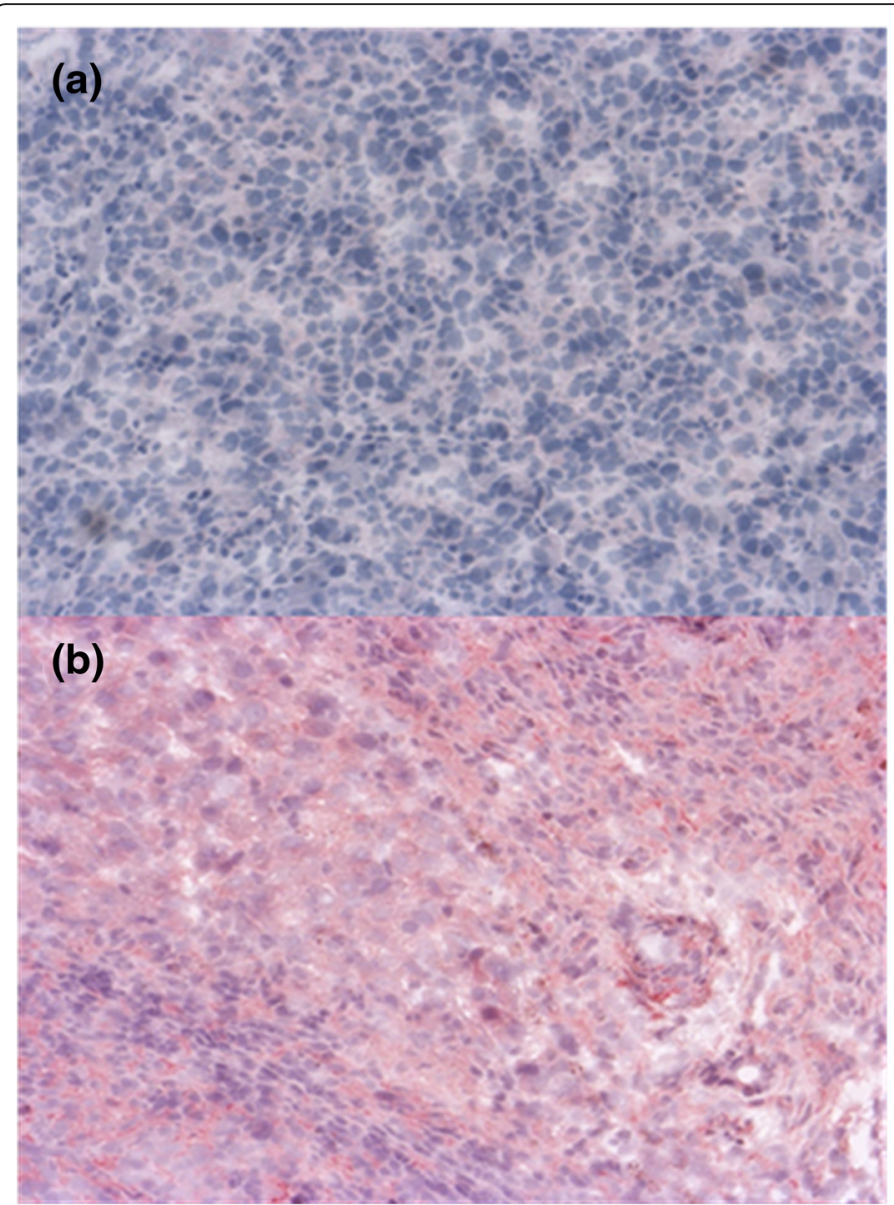

(c)

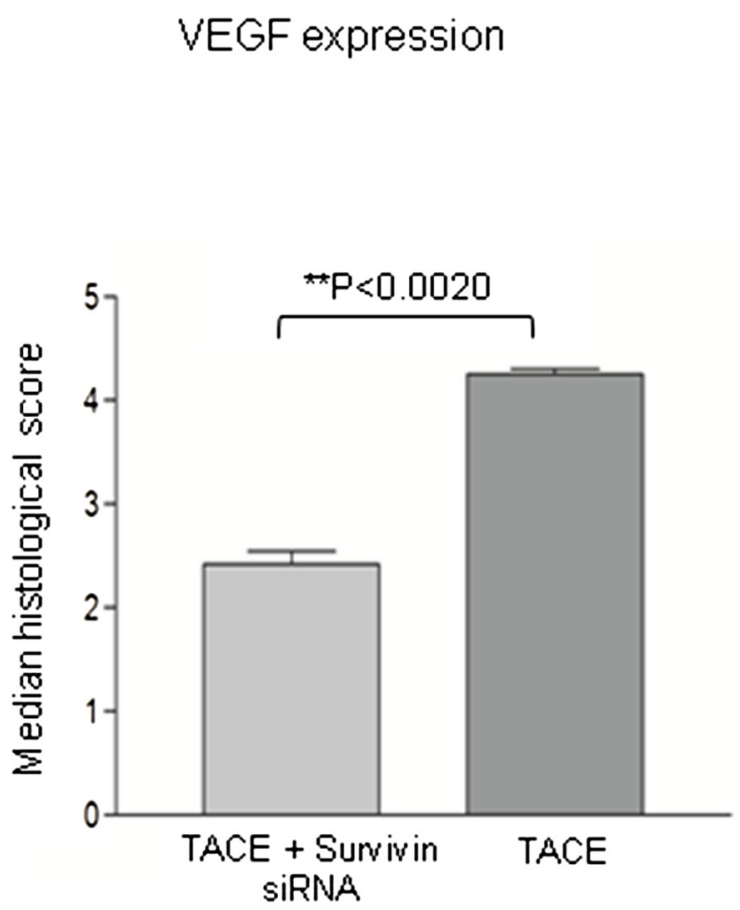

Fig. 3 The immunohistochemical staining of VEGF in hepatocellular carcinoma. a VEGF staining in hepatocellular carcinoma in the group A (TACE + survivin siRNA) $(\times 100)$. b Significantly higher VEGF staining in hepatocellular carcinoma was observed in group B (control group, TACE alone) than group A $(\times 100)$. c Median histological score of VEGF $(N=10)$

potent for gene silencing, making it attractive tool for silencing of target genes in cancer [24]. An Adenovirusmediated siRNA expression vector was reported to decrease Survivin expression of the established HCC tumor in nude mice. In vitro study showed that stable Survivin-knockdown inhibited cancer cell proliferation, enhanced apoptotic susceptibility, arrested cell cycle in the G1 phase and resulted in an apparent mitotic catastrophe. An additional in vivo study showed that intratumoral injection of Adenovirus-delivered Survivin siRNA suppressed tumor growth by spontaneous apoptosis of cancer cells and significantly prolonged animal survival [25]. However, in vivo systemic delivery of siRNA-based therapeutics to tumor tissues/cells remains a challenge. The major limitations against the use of siRNA as a therapeutic tool are its degradation by serum nucleases, poor cellular uptake, nonspecific immune stimulation and rapid renal clearance following systemic administration [24, 26].

The current study was designed to reduce tumor progression by using a combination of transarterial administration of Survivin siRNA and TACE using the sandwich technique in an animal model of HCC. We did not include intravenous delivery approach because the transarterial route was much more efficient in the treatment of HCC. In the current study however we did not evaluate the expression of Survivin directly in the Hepatoma 3924a in addition we did not assess the degree of suppression of Survivin expression in response to treatment for several reasons. First the expression of Survivin has been reported to occur in several HCC cell lines and in human HCC tissue [27], it has been reported to be highly expressed in the vast majority of human cancers including HCC [28]. Second we relied on the radiologic oncologic assessment of the therapeutic effect of Survivin siRNA rather than on its direct assessment. A direct assessment of the suppression of Survivin was rather outside the scope of the current study. Finally Survivin expression has been shown to significantly correlate with VEGF expression in HCC [29], hence we relied on this correlation to indirectly assess Survivin suppression (through VEGF assessment). Still we regard this as one 
of the limitations of the study and recommend the subject for further evaluation in future studies assessing not only the response based on radiologic evidence but based on direct assessment of Survivin. Another limitation of the current study is the lack of a negative control group (receiving si-control RNA).

Survivin has been shown to play an important role in the regulation of expression of VEGF in breast cancer lymphatic metastases [30]. In addition it has been shown to significantly correlate with the expression with VEGF in hepatocellular carcinoma and promote the expression of VEGF $[28,29]$. Our experimental results demonstrated that groups A (TACE + Survivin siRNA) showed a significant reduction of tumor growth in the period of observation compared to the control group (TACE alone). Higher Immunohistochemical expression of VEGF in hepatocellular carcinoma was observed in group B (TACE alone) than that of group A (TACE + Survivin siRNA) $(P<0.01)$. The invasive progression of tumor cells in group A was noticeably inhibited compared with group B. This complex targeting treatment method seemed to overcome the insufficiency of TACE and improve the overall therapeutic effects.

\section{Conclusion}

In conclusion, the additional injection of Survivin siRNA to the routine protocol of TACE has shown to be safe and effective in an animal model of HCC in rats. This combined therapeutic therapy noticeably inhibited the growth of the hepatic carcinoma in rats compared with TACE alone. Further experimental studies will be required to fully understand the benefits and risks of this strategy for treating HCC before transferring it to human studies.

\section{Ethics approval and consent to participate}

The current animal study was approved by the Regional Administrative Authority in Darmstadt, Germany.

\section{Consent for publication}

The current study does not contain any personal materials that require patient approval.

\section{Availability of data and materials}

Due to organizational restrictions the data will not be available.

\section{Abbreviations \\ HCC: hepatocellular carcinoma; SDS-PAGE: sodium dodecyl sulfate polyacrylamide gel electrophoresis; siRNA: small interfering RNA; TACE: transarterial chemoembolization; VEGF: vascular endothelial growth factor expression.}

\section{Competing interests}

The authors declare that they have no competing interests.

\section{Authors' contributions}

Study concept and design: all authors. Experimental animal study: JQ, UI, AT, YH, HK. Data collection and analysis: EO, JQ, UI, YH, AT. Literature Research: TJV, EO, WOB, NAN, TG, RH, NNN. Manuscript editing and preparation: all authors. Statistical analysis: JQ, AT. Acceptance of the final version: all authors. All authors read and approved the final manuscript.

\section{Acknowledgment}

Neither the authors nor the study received any form of grant support.

\section{Author details}

${ }^{1}$ Institute for Diagnostic and Interventional Radiology, Johann Wolfgang Goethe-University, Theodor-Stern-Kai 7, Frankfurt 60590, Germany.

${ }^{2}$ Department of General Surgery, Johann Wolfgang Goethe-University, Frankfurt, Germany. ${ }^{3}$ Department of Diagnostic and Interventional Radiology, Faculty of Medicine, Cairo University, Cairo, Egypt. ${ }^{4}$ Department of Diagnostic and Interventional Radiology, Faculty of Medicine, Alexandria University, Alexandria, Egypt.

Received: 19 September 2015 Accepted: 11 May 2016

Published online: 23 May 2016

\section{References}

1. Ferlay J, Shin H-R, Bray F, Forman D, Mathers C, Parkin DM. Estimates of worldwide burden of cancer in 2008: GLOBOCAN 2008. Int J Cancer. 2010; 127(12):2893-917.

2. Qian J, Feng GS, Vogl T. Combined interventional therapies of hepatocellular carcinoma. World J Gastroenterol. 2003;9(9):1885-91.

3. Church DN, Talbot DC. Survivin in solid tumors: rationale for development of inhibitors. Curr Oncol Rep. 2012;14:120-8.

4. Sah NK, Khan Z, Khan GJ, Bisen PS. Structural, functional and therapeutic biology of survivin. Cancer Lett. 2006:244(2):164-71.

5. Fukuda S, Pelus LM. Survivin, a cancer target with an emerging role in normal adult tissues. Mol Cancer Ther. 2006;5(5):1087-98.

6. Fukuda S, Pelus LM. Regulation of the inhibitor-of-apoptosis family member survivin in normal cord blood and bone marrow CD34(+) cells by hematopoietic growth factors: implication of survivin expression in normal hematopoiesis. Blood. 2001;98(7):2091-100.

7. Capalbo G, Rodel C, Stauber RH, Knauer SK, Bache M, Kappler M, Rodel F. The role of survivin for radiation therapy. Prognostic and predictive factor and therapeutic target. Strahlenther Onkol. 2007;183(11):593-9.

8. Kappler M, Bache M, Bartel F, Kotzsch M, Panian M, Würl P, Blümke K, Schmidt H, Meye A, Taubert H. Knockdown of survivin expression by small interfering RNA reduces the clonogenic survival of human sarcoma cell lines independently of p53. Cancer Gene Ther. 2004;11(3):186-93.

9. Geschwind J-FH, Ramsey DE, Choti MA, Thuluvath PJ, Huncharek MS. Chemoembolization of hepatocellular carcinoma: results of a metaanalysis. Am J Clin Oncol. 2003;26(4):344-9.

10. Vogl TJ, Naguib NN, Nour-Eldin NE, Rao P, Emami AH, Zangos S, Nabil M, Abdelkader A. Review on transarterial chemoembolization in hepatocellular carcinoma: palliative, combined, neoadjuvant, bridging, and symptomatic indications. Eur J Radiol. 2009;72(3):505-16.

11. Yang $X$, Hong H, Grailer JJ, Rowland IJ, Javadi A, Hurley SA, Xiao Y, Yang $Y$, Zhang $Y$, Nickles RJ, et al. CRGD-functionalized, DOX-conjugated, and 64CUlabeled superparamagnetic iron oxide nanoparticles for targeted anticancer drug delivery and PET/MR imaging. Biomaterials. 2011;32(17):4151-60.

12. Qian J, Truebenbach J, Graepler F, Pereira P, Huppert P, Eul T, Wiemann G, Claussen C. Application of poly-lactide-co-glycolide-microspheres in the transarterial chemoembolization in an animal model of hepatocellular carcinoma. World J Gastroenterol. 2003;9(1):94-8.

13. Carlsson G, Gullberg B, Hafström L. Estimation of liver tumor volume using different formulas?An experimental study in rats. J Cancer Res Clin Oncol. 1983;105(1):20-3.

14. Achenbach $T$, Seifert JK, Pitton MB, Schunk $K$, Junginger $T$. Chemoembolization for primary liver cancer. Eur J Surg Oncol (EJSO). 2002;28(1):37-41.

15. Kobayashi N, Ishii M, Ueno Y, Kisara N, Chida N, Iwasaki T, Toyota T. Co-expression of $\mathrm{BCl}-2$ protein and vascular endothelial growth factor in hepatocellular carcinomas treated by chemoembolization. Liver Int. 1999;19(1):25-31. 
16. Kawasaki H, Toyoda M, Shinohara H, Okuda J, Watanabe I, Yamamoto T, Tanaka K, Tenjo T, Tanigawa N. Expression of survivin correlates with apoptosis, proliferation, and angiogenesis during human colorectal tumorigenesis. Cancer. 2001;91(11):2026-32.

17. Hayashi N, Asano K, Suzuki H, Yamamoto T, Tanigawa N, Egawa S, Manome Y. Adenoviral infection of survivin antisense sensitizes prostate cancer cells to etoposide in vivo. Prostate. 2005;65(1):10-9.

18. Rodel F, Hoffmann J, Grabenbauer GG, Papadopoulos T, Weiss C, Gunther K, Schick C, Sauer R, Rodel C. High survivin expression is associated with reduced apoptosis in rectal cancer and may predict disease-free survival after preoperative radiochemotherapy and surgical resection. Strahlenther Onkol. 2002;178(8):426-35.

19. Bache M, Holzapfel D, Kappler M, Holzhausen HJ, Taubert H, Dunst J, Hansgen G. Survivin protein expression and hypoxia in advanced cervical carcinoma of patients treated by radiotherapy. Gynecol Oncol. 2007;104(1): 139-44.

20. Yang W, Sun T, Cao J, Liu F. Survivin downregulation by siRNA/cationic liposome complex radiosensitises human hepatoma cells in vitro and in vivo. Int J Radiat Biol. 2010;86(6):445-57.

21. Chantalat L, Skoufias DA, Kleman J-P, Jung B, Dideberg O, Margolis RL. Crystal structure of human Survivin reveals a bow tie-shaped dimer with two unusual a-helical extensions. Mol Cell. 2000;6(1):183-9.

22. Ryan BM, O'Donovan N, Duffy MJ. Survivin: a new target for anti-cancer therapy. Cancer Treat Rev. 2009;35(7):553-62.

23. Oh Y-K, Park TG. siRNA delivery systems for cancer treatment. Adv Drug Deliv Rev. 2009:61(10):850-62

24. Zhang R, Ma L, Zheng M, Ren J, Wang T, Meng Y, Zhao J, Jia L, Yao L, Han $\mathrm{H}$, et al. Survivin knockdown by short hairpin RNA abrogates the growth of human hepatocellular carcinoma xenografts in nude mice. Cancer Gene Ther. 2009;17(4):275-88.

25. Ozpolat B, Sood AK, Lopez-Berestein G. Nanomedicine based approaches for the delivery of siRNA in cancer. J Intern Med. 2010;267(1):44-53.

26. Leung RKM, Whittaker PA. RNA interference: from gene silencing to genespecific therapeutics. Pharmacol Ther. 2005;107(2):222-39.

27. Ito T, Shiraki K, Sugimoto K, Yamanaka T, Fujikawa K, Ito M, Takase K, Moriyama M, Kawano H, Hayashida M, et al. Survivin promotes cell proliferation in human hepatocellular carcinoma. Hepatology. 2000;31(5): 1080-5.

28. Su C. Survivin in survival of hepatocellular carcinoma. Cancer Lett. 2015. doi: 10.1016/j.canlet.2015.06.016.

29. Zhu H, Chen XP, Zhang WG, Luo SF, Zhang BX. Expression and significance of new inhibitor of apoptosis protein survivin in hepatocellular carcinoma. World J Gastroenterol. 2005;11(25):3855-9.

30. Cai X, Ma S, Gu M, Zu C, Qu W, Zheng X. Survivin regulates the expression of VEGF-C in lymphatic metastasis of breast cancer. Diagn Pathol. 2012;7:52.

\section{Submit your next manuscript to BioMed Central and we will help you at every step:}

- We accept pre-submission inquiries

- Our selector tool helps you to find the most relevant journal

- We provide round the clock customer support

- Convenient online submission

- Thorough peer review

- Inclusion in PubMed and all major indexing services

- Maximum visibility for your research

Submit your manuscript at www.biomedcentral.com/submit

) Biomed Central 